\title{
FATORES RELACIONADOS COM AS REINTERNAÇÕES DE PORTADORES DE ESQUIZOFRENIA
}

Tânia de Lázari Sanches Pinheiro¹, Luiza Helena de Oliveira Cazola², Cibele de Moura Sales³, Ana Rachel Oliveira de Andrade ${ }^{4}$

RESUMO: Este estudo de caráter descritivo objetivou identificar os fatores envolvidos nas reinternações dos portadores de esquizofrenia de um hospital psiquiátrico em uma capital brasileira. Os sujeitos da pesquisa foram portadores de esquizofrenia com histórico de três ou mais internações na instituição, no período de um ano. Dados secundários foram coletados com pesquisa documental e os dados primários com entrevistas com os responsáveis pelos pacientes. Como fatores relacionados às reinternações estão a não-continuidade do cuidado ao portador de esquizofrenia na rede de atenção substitutiva, após a alta hospitalar, a baixa condição socioeconômica dos pacientes e familiares, as dificuldade dos familiares em lidar e conviver com os adoecidos, a não-adesão ao tratamento medicamentoso, e a gravidade da doença. Conclui-se que são necessárias estratégias para a corresponsabilização da família no acompanhamento ao paciente, assim como melhor assistência das instituições complementares ao tratamento hospitalar, possibilitando redução nas reinternações.

PALAVRAS-CHAVE: Esquizofrenia; Serviços de saúde mental; Readmissão do paciente.

\section{FACTORS RELATED WITH READMISSIONS OF SCHIZOPHRENIC PATIENTS}

ABSTRACT: This descriptive study aimed to identify factors involved in readmissions of schizophrenic patients in a psychiatric hospital in a Brazilian capital. The research subjects were schizophrenic patients with a history of three or more admissions in the institution within one year. Secondary data were collected through documentary research and primary data through interviews with parents or caregivers. As readmissions-related factors are: the discontinuance of care for patients with schizophrenia in the care network replacement after hospital discharge, low socioeconomic status of patients and families, the difficulty of families to cope and live with the ill, non-compliance to drug therapy and disease severity. It was concluded that strategies are needed for the joint responsibility of the family in monitoring the patient, as well as a better care performed by the complementary institutions after hospital treatment, enabling a reduction in readmissions.

KEYWORDS: Schizophrenia; Mental health services; Patient readmission.

\section{FACTORES RELACIONADOS CON LAS REINTERNACIONES DE PORTADORES DE ESQUIZOFRENIA}

RESUMEN: Este estudio de carácter descriptivo objetivó identificar los factores envueltos en las reinternaciones de los portadores de esquizofrenia de un hospital psiquiátrico en una capital brasileira. Los sujetos de la investigación fueron portadores de esquizofrenia con histórico de tres o más internaciones en la institución, en el período de un año. Datos secundarios fueron recogidos con investigación documental y los datos primarios con entrevistas con los responsables por los pacientes. Como factores relacionados a las reinternaciones están la no-continuidad del cuidado al portador de esquizofrenia en la red de atención substitutiva, después del alta hospitalario, la baja condición socioeconómica de los pacientes y familiares, las dificultades de los familiares en lidiar y convivir con los adolecidos, la no-adhesión al tratamiento medicamentoso, y la gravedad de la enfermedad. Conclúyese que son necesarias estrategias para la co-responsabilización de la familia en el acompañamiento al paciente, así como mejor atención de las instituciones complementares al tratamiento hospitalario, posibilitando reducción en las reinternaciones.

PALABRAS CLAVE: Esquizofrenia; Servicios de salud mental; Readmisión del paciente.

\footnotetext{
${ }^{1}$ Terapeuta Ocupacional do Hospital Nosso Lar, Campo Grande-MS.

${ }^{2}$ Enfermeira. Mestre em Saúde Coletiva. Doutoranda em Saúde e Desenvolvimento na Região Centro-Oeste da Universidade Federal de Mato Grosso do Sul-UFMS. Professora Auxiliar da UFMS.

${ }^{3}$ Enfermeira. Doutora em Ciências da Saúde. Docente da Universidade Estadual de Mato Grosso do Sul-UEMS.

${ }^{4}$ Bióloga. Mestre em Meio Ambiente e Desenvolvimento Regional. Doutoranda do Programa de Pós-Graduação em Doenças InfectoContagiosas da UFMS.
}

Autor correspondente:

Tânia de Lázari Sanches Pinheiro

Hospital Nosso Lar

Rua Tijuca, 240 - 79110-280 - Campo Grande-MS, Brasil

Recebido: 23/09/09

E-mail: tanialsp@hotmail.com

Aprovado: 30/04/10

Cogitare Enferm. 2010 Abr/Jun; 15(2):302-7 


\section{INTRODUÇÃO}

A Reforma Psiquiátrica é o processo histórico de reformulação crítica e prática do modelo clássico e do paradigma da psiquiatria. Isso porque o espaço dedicado ao cuidado com os portadores de transtorno mental, o hospital psiquiátrico, anteriormente denominado manicômio, revelou-se como lugar de segregação e controle, fundamentando-se nos processos de exclusão social e na periculosidade: lugar do louco, do improdutivo, daquele que se desencaixa da ordem, da razão e da produção ${ }^{(1)}$. A crítica realizada pelo movimento de Reforma é tanto conjuntural ao subsistema nacional de Saúde Mental, quanto direcionada ao saber e às instituições psiquiátricas clássicas ${ }^{(2)}$.

O processo de Reforma Psiquiátrica brasileira data de pouco mais de 20 anos e tem como marca distintiva e fundamental a reivindicação da cidadania do insano ${ }^{(3)}$. No contexto do movimento da Reforma Psiquiátrica, surgiram novas formas de assistência e intervenção ao portador de transtorno mental, os serviços substitutivos, tendo como um de seus principais objetivos a desconstrução do modelo de atenção hospitalocêntrico.

A trajetória da desinstitucionalização é caracterizada pelo nascimento de novos serviços e estratégias, como os Centros de Atenção Psicossocial (CAPS). Os CAPS, de acordo com a definição do Ministério de Saúde, constituem-se em serviços de saúde abertos e comunitários do Sistema Único de Saúde (SUS). Estes serviços são locais de referência e tratamento para os que sofrem de transtornos mentais: psicoses, neuroses graves e outros quadros correlatos, cuja severidade ou persistência justifiquem sua permanência num dispositivo de cuidado intensivo, comunitário, personalizado e promotor de vida. Eles têm o objetivo de oferecer atendimento à população de sua área de abrangência, efetuando o acompanhamento clínico e a reinserção social dos usuários por meio do acesso ao lazer, do exercício dos direitos civis e do fortalecimento dos laços familiares e comunitários, e precisam estar integrados à rede de atenção à saúde ${ }^{(4)}$.

Apesar dos serviços substitutivos ao hospital psiquiátrico estarem em processo avançado de implantação, há grandes desafios dado a complexidade de desconstrução de modelo de atenção, que inclui mudanças nos profissionais de saúde, no olhar, nas ações e nos serviços. Ainda, impõe-se a necessidade do cuidado à saúde mental estar integrado na e com a rede de atenção à saúde nos mais diversos níveis de complexidade. Enfim, o desafio posto é a diminuição de reinternações, em hospitais psiquiátricos, de portadores de transtorno mental.

Este estudo teve por objetivo identificar os fatores envolvidos nas reinternações dos portadores de esquizofrenia de um hospital psiquiátrico localizado em uma capital brasileira a qual possui serviços substitutivos implantados.

Reinternação, neste estudo, é considerado como o conjunto de três ou mais internações ocorridas no período de um ano. Foram considerados pacientes esquizofrênicos os que tinham o registro no prontuário do diagnóstico classificado como F20 do Código Internacional de Doenças (CID 10) ${ }^{(5)}$.

A esquizofrenia, segundo a classificação da Organização Mundial da Saúde, inclui os sintomas de primeira ordem: ouvir os próprios pensamentos soando alto; escutar vozes sob a forma de argumento e contraargumento; escutar, com comentários, vozes que acompanham as próprias atividades; ter vivências de influência corporal; ter roubo de pensamento e outras formas de influência de pensamento; sentir tudo como sendo feito ou influenciado pelos outros no campo dos sentimentos, pulsões e vontade; ter percepção delirante $\mathrm{e}^{(5)}$.

O diagnóstico de esquizofrenia foi o escolhido porque, dos pacientes que sofrem sucessivas reinternações na instituição estudada, quase a totalidade são portadores desta doença. Acredita-se que este estudo possa oferecer aos serviços de saúde instrumentos e/ou conhecimentos necessários para a reflexão da prática no campo da Saúde Mental e para o planejamento de ações que contribuam para a diminuição de reinternações em hospitais psiquiátricos.

\section{PROCEDIMENTOS METODOLÓGICOS}

Este estudo foi realizado em um hospital psiquiátrico localizado em Campo Grande, Estado do Mato Grosso do Sul com serviços substitutivos implantados. Este hospital dispõe de 160 leitos para o SUS e é referência para o estado.

Foram incluídos no estudo 32 portadores de esquizofrenia que atenderam aos seguintes critérios de inclusão: apresentar registro em prontuário de três ou mais internações na instituição, no período de um ano, e residir na mesma cidade do hospital, por esta possuir os serviços substitutivos implantados.

Para este grupo, foram identificados nos prontuários os seguintes dados: características socioeconômi- 
cas, diagnóstico, antecedentes familiares, início da doença, comorbidades, sintomas clínicos, atividades laborativas e frequência em serviços substitutivos. Realizou-se a pesquisa documental nos arquivos da instituição (prontuários e relatórios) e os dados foram tabulados e submetidos à estatística descritiva.

Para os familiares de pacientes que possuíam quatro ou mais internações em um ano, foram realizadas entrevistas com questões abertas, sendo que apenas quatro pacientes atenderam a esse critério de inclusão. Buscou-se, através dessas entrevistas, conhecer os fatores envolvidos nas reinternações, segundo a ótica dos seus familiares.

Considerou-se responsável o acompanhante do paciente no momento da internação, sendo na totalidade familiares. Para a aplicação do instrumento de coleta de dados realizou-se contato prévio com o responsável, via telefone, para agendamento da entrevista, que foi realizada em seu domicílio.

O instrumento de coleta de dados foi composto por três grupos de variáveis: comportamento do paciente no período de alta hospitalar, indicadores de necessidade de reinternação e serviços de saúde frequentados.

Os dados primários obtidos das questões do instrumento foram agrupados em categorias assemelhadas e as respostas quantificadas de acordo com a frequência com que foram mencionadas.

Em atendimento ao disposto na Resolução n. 196/96, foi preservado o anonimato dos sujeitos da pesquisa, bem como o sigilo das informações coletadas. Para a realização das entrevistas foi elaborado o Termo de Consentimento Livre e Esclarecido, assinado em duas vias, ficando uma com o entrevistado e outra com o pesquisador. O estudo foi aprovado pelo Comitê de Ética em Pesquisa da Universidade Federal de Mato Grosso do Sul, sob o Protocolo n. 708.

\section{RESULTADOS E DISCUSSÃO}

A esquizofrenia acomete pessoas em idades precoces, conduzindo a alterações graves do pensamento, afeto e vontade. Essas apresentam afastamento da realidade externa, com enorme desgaste emocional e econômico para si, suas famílias e sociedade. Apesar dos grandes avanços da bioquímica, genética e terapêutica psicofarmacológica, ainda não se tem comprovação científica da etiologia biológica da esquizofrenia, embora as observações empíricas apontem nesta direção. Sabe-se, entretanto, que fatores biopsicossociais influenciam o início, o tratamento, as recaídas e o prognóstico, alterando todo o curso da enfermidade,acometendo igualmente ambos os $\operatorname{sexos}^{(6)}$.

Em relação ao início dos sintomas, eles podem ocorrer entre 15 e 25 anos em homens e 25 e 35 anos em mulheres ${ }^{(6)}$. Neste estudo não foi possível observar essa diferença, pois tanto homens quanto mulheres tiveram, em sua maioria, o início da doença até 25 anos (Tabela 1).

Quanto mais precocemente se iniciam os sintomas, maiores os danos na vida socioafetiva, na gravidade dos sintomas e na evolução do quadro

Tabela 1 - Início dos sintomas de portadores de esquizofrenia de uma instituição psiquiátrica, segundo o sexo e a idade. Campo Grande, MS.

\begin{tabular}{lcccccc}
\hline & \multicolumn{2}{c}{ Homens } & \multicolumn{2}{c}{ Mulheres } & \multicolumn{2}{c}{ Total } \\
\cline { 2 - 7 } Faixa etária & $\mathbf{N}$ & $\mathbf{\%}$ & $\mathbf{N}$ & $\mathbf{\%}$ & $\mathbf{N}$ & $\mathbf{\%}$ \\
\hline $15-20$ anos & 10 & 50,00 & 7 & 58,34 & 17 & 53,12 \\
$20-25$ anos & 5 & 25,00 & 3 & 25,00 & 8 & 25,00 \\
Acima de 25 anos & 0 & 0,00 & 1 & 8,33 & 1 & 3,12 \\
Sem informação & 5 & 25,00 & 1 & 8,33 & 6 & 18,76 \\
\hline Total & $\mathbf{2 0}$ & $\mathbf{1 0 0 , 0 0}$ & $\mathbf{1 2}$ & $\mathbf{1 0 0 , 0 0}$ & $\mathbf{3 2}$ & $\mathbf{1 0 0 , 0 0}$ \\
\hline
\end{tabular}

patológico. Um dos problemas acarretados pelo início precoce da esquizofrenia é a dificuldade de seu portador em vivenciar uma união afetiva satisfatória, devido aos sintomas ${ }^{(7-8)}$.

Pessoas acometidas por esse transtorno mental em idade precoce possuem dificuldades em manter um relacionamento afetivo estável e de se $\operatorname{casar}^{(8)}$. Neste estudo, observou-se que a maioria dos portadores de esquizofrenia eram solteiros, um total de $81,26 \%$.

Como os sintomas da esquizofrenia podem ter início ainda na adolescência, muitos pacientes não conseguem completar os estudos de escola fundamental ou média, podendo isso decorrer tanto dos períodos de internação quanto daqueles passados em crise, o que impede a regularidade na frequência escolar, diminuindo, posteriormente, a possibilidade de emprego e de ascensão profissional. No estudo, observou-se baixo nível de escolaridade (Tabela 2), o qual se mostra como um dos fatores que impossibilitam o exercício de atividades laborativas e o sustento (Tabela 3).

As frequentes hospitalizações podem também contribuir para o processo de cronificação da doença, aumentando o isolamento e distanciamento do mundo 
Tabela 2 - Portadores de esquizofrenia de uma instituição psiquiátrica, segundo a escolaridade. Campo Grande, MS.

\begin{tabular}{lcc}
\hline Escolaridade & $\mathbf{N}$ & $\mathbf{\%}$ \\
\hline Analfabetos & 2 & 6,25 \\
Até $6 .^{\text {a }}$ série & 15 & 46,88 \\
Fundamental completo & 3 & 9,37 \\
Médio completo & 4 & 12,50 \\
Superior incompleto & 1 & 3,13 \\
Sem informação & 7 & 21,87 \\
\hline Total & $\mathbf{3 2}$ & $\mathbf{1 0 0 , 0 0}$ \\
\hline
\end{tabular}

Tabela 3 - Portadores de esquizofrenia de uma instituição psiquiátrica, segundo o exercício de atividade laborativa. Campo Grande, MS.

\begin{tabular}{lcc}
\hline Exercício de atividade laborativa & $\mathbf{N}$ & $\mathbf{\%}$ \\
\hline Exerce & 1 & 3,12 \\
Não exerce devido ao transtorno mental & 14 & 43,75 \\
Nunca exerceu & 9 & 28,13 \\
Sem informação & 8 & 25,00 \\
\hline Total & $\mathbf{3 2}$ & $\mathbf{1 0 0 , 0 0}$ \\
\hline
\end{tabular}

real, privação do convívio familiar e social e promoção do estigma de doente mental. Dos portadores de esquizofrenia, 93,75\% eram doentes crônicos e 59,38\% tinham esse distúrbio há mais de cinco anos (Tabela 4).

Sobressaíram na pesquisa sintomas considerados graves e incapacitantes, sobretudo nas esferas social,

Tabela 4 - Portadores de esquizofrenia de uma instituição psiquiátrica, segundo a cronicidade e o tempo decorrido desde o adoecimento. Campo Grande, MS.

\begin{tabular}{lcc}
\hline Variáveis & $\mathbf{N}$ & $\mathbf{\%}$ \\
\hline Cronicidade & 30 & 93,75 \\
Sim & 2 & 6,25 \\
Não & & \\
Tempo de adoecimento & 2 & 6,25 \\
Até 2 anos & 6 & 18,75 \\
2-5 anos & 3 & 9,38 \\
5-10 anos & 9 & 28,12 \\
10-20 anos & 4 & 12,50 \\
20-30 anos & 3 & 9,38 \\
Mais de 30 anos & 5 & 15,62 \\
Sem informação & &
\end{tabular}

afetiva e ocupacional, e que podem vir acompanhados de alterações de comportamento que agravam o distanciamento social, levando à dificuldade de ressocialização. Os principais sintomas apresentados pelos portadores de esquizofrenia no momento da internação estão apresentados na Tabela 5.

Foram consideradas como alterações de comportamento (Tabela 5) a agressividade verbal, heteroagres-

Tabela 5 - Portadores de esquizofrenia de uma instituição psiquiátrica, segundo os sintomas apresentados. Campo Grande, MS.

\begin{tabular}{lcc}
\hline Sintomas* & N & \% \\
\hline Delírios & 29 & 90,62 \\
Alucinações & 20 & 62,50 \\
Embotamento afetivo & 11 & 34,37 \\
Alterações do pensamento & 24 & 75,00 \\
Autonegligência & 22 & 68,75 \\
Alterações de comportamento & 32 & 100,00 \\
\hline *Mais de uma resposta possível & &
\end{tabular}

sividade, impulsividade, agitação, irritabilidade, deambulação sem destino, hostilidade, atitudes bizarras, condutas sociais inadequadas e perda de pudor.

Verificou-se também que $75 \%$ dos portadores de esquizofrenia não faziam uso correto da medicação, quando da entrada no hospital. Cabe lembrar, porém, que a administração correta da medicação não previne totalmente a reagudização dos sintomas.

Como indicadores de bom prognóstico para portadores de esquizofrenia considera-se o diagnóstico precoce e o tratamento apropriado, sendo que este último inclui a adesão a um regime medicamentoso adequado, adesão essa que não se verificou na maioria dos pesquisados. Este achado pode decorrer, de acordo com o estudo, dos efeitos colaterais provocados pela medicação (segundo os próprios adoecidos), da dificuldade de seus familiares em obter a medicação nos locais indicados e especializados, por não possuírem condições financeiras de adquiri-los, ou mesmo da falta de cuidador que administre a medicação corretamente ${ }^{(9)}$. Sabe-se, contudo, que há disponibilização gratuita de medicamentos nos CAPS e mesmos os de alto custo encontram-se disponíveis no Brasil por meio do protocolo de esquizofrenia refratária ${ }^{(10-11)}$.

Outra razão para a falta de adesão ao tratamento é que a maioria dos portadores de esquizofrenia 
tem insight pobre em relação ao fato de que sofrem de uma doença psicótica. As evidências sugerem ser uma manifestação da doença em si, em vez de uma estratégia de enfrentamento ${ }^{(12)}$.

Em relação à frequência destes usuários a outros serviços e atendimentos em Saúde Mental, que não os disponíveis em regime de internação, apenas um $(3,12 \%)$ referiu frequentar.

Os portadores de transtorno mental após receberem alta hospitalar necessitam de tratamento adequado e de intervenção e atenção voltadas, principalmente, à reinserção na comunidade. Isso deve ser alcançado, em especial, com serviços substitutivos, cujo foco de tratamento seja o indivíduo e não a doença. Identificar novas estratégias de encaminhamento e integração em serviços substitutivos torna-se fundamental, visto que a entrada dos portadores de transtornos mentais nesses serviços pode conduzir a benefícios relacionados à adesão à terapêutica medicamentosa de manutenção e a novas redes de interação, aumentando seus contatos e relacionamentos sociais, e a participação ativa da família ${ }^{(13)}$.

As causas mais frequentes das reinternações, segundo a ótica dos familiares foram: nervosismo, agressividade, insônia, fugas de casa, episódios de violência, delírios, risco de vida para si, perda do pudor, irritação e agitação psicomotora.

Com relação ao nível de conhecimento quanto à existência de outros locais ou serviços para tratamento que não o hospital psiquiátrico, apenas um responsável, sabia da existência destes serviços substitutivos.

Alguns familiares percebem a internação como um período de alívio, por não saberem como agir frente a comportamentos inesperados e bizarros. Observouse que a totalidade apresentou dificuldades quanto ao controle da ingestão de medicamento por parte de seu familiar adoecido.

Publicação refere que os familiares "perdem o chão", ficam paralisados quando alguém alucina. Não sabem se confrontam, brigam ou colocam limites ${ }^{(14)}$. Afirma-se, ainda, que os familiares sentem-se incapazes em alguns momentos de se relacionar com o adoecido, atribuindo essa atitude ao fato de desconhecerem a doença (o que faz com que atividades rotineiras tornemse impraticáveis) e mostrando-se ineficazes para atender a qualquer situação cotidiana ${ }^{(15)}$. E não são apenas os familiares que se sentem incapazes de atender o portador de transtorno mental, pois muitas vezes até a própria equipe de saúde não se sente capacitada para assistir o doente e seus familiares ${ }^{(16)}$.
Identificou-se, ainda, a falta de conhecimento e entendimento sobre a esquizofrenia e também a necessidade de suporte emocional para si mesmo.

\section{CONCLUSÃO}

Entre os fatores envolvidos nas reinternações está a falta de conhecimento pelos familiares da existência da rede de serviços substitutivos ao hospital. O baixo nível socioeconômico dos pacientes, o não seguimento correto do tratamento medicamentoso, bem como falhas do familiar no controle de ingestão do medicamento, também são fatores que contribuem para a reinternação.

O desconhecimento sobre a patologia e as dificuldades dos familiares em conviver e lidar com esses adoecidos faz com que procurem na internação solução para a problemática. Dessa forma, torna-se necessário uma intervenção junto aos familiares para que possam lidar com a doença e com a discriminação e incompreensão sociais.

\section{REFERÊNCIAS}

1. Nicácio MF. Da instituição negada à instituição inventada. In: Lancetti A, coordenador. Saúde Loucura. São Paulo: Hucitec; 1989. p. 91-108.

2. Amarante P. Loucos pela vida: a trajetória da reforma psiquiátrica no Brasil. Rio de Janeiro: Fiocruz;1995.

3. Tenório F. A reforma psiquiátrica brasileira da década de 1980 aos dias atuais, história e conceitos. Hist Cienc Saúde Manguinhos 2000;9(1):25-59.

4. Ministério da Saúde (BR). Saúde mental no SUS: os centros de atenção psicossocial. Brasília: Ministério da Saúde; 2004.

5. Organização Mundial da Saúde. Classificação dos transtornos mentais e do comportamento da CID 10. Porto Alegre: Artes Médicas; 1993.

6. Nunes EPF, Bueno JR, Nardi AE. Psiquiatria e saúde mental: conceitos clínicos e terapêuticos fundamentais. São Paulo: Atheneu; 2001.

7. Tostes LRM, Moraes LRN. Esquizofrenia: curso, evolução e prognóstico. J Bras Psiquiatr. 1989;38(4):233-9.

8. Chaves AC. Diferença entre os sexos na esquizofrenia.In: Shirakawa I, Mari JJ, Chaves AC, editores. O desafio da esquizofrenia. São Paulo: Lemos; 1998. p. 85-97. 
9. Sá Júnior LSM. Fundamentos de psicopatologia: bases do exame psíquico. São Paulo: Atheneu; 1988.

10. Candiago RH, Abreu PB. Uso do Datasus para avaliação dos padrões das internações psiquiátricas, Rio Grande do Sul. Rev Saúde Pública. 2007;41(5):821-9.

11. Cardoso C, Seminotti N. The psychoterapeutic group in the CAPS. Cien Saúde Colet. 2006;11(3):775-83.

12. American Psychiatric Association. Manual diagnóstico e estatístico de transtornos mentais DSM- IV-TR. Trad. de Cláudia D. Porto Alegre: Artmed; 2003.

13. Salles MM, Barros S. Reinternação em hospital psiquiátrico: a compreensão do processo saúde/doença na vivência do cotidiano. Rev Esc Enferm USP. 2007;41(1):73-81.

14. Melman J. Família e doença mental: repensando as relações entre profissionais de saúde e familiares. São Paulo: Escrituras; 2001.

15. Reinaldo MAS, Saeki T. O itinerário terapêutico em saúde mental pela história oral de vida de um paciente psiquiátrico. In: Souza MCBM, Costa MCS, organizadores. Saúde mental numa sociedade em mudança. Ribeirão Preto:Legis Summa; 2005. p.167-81.

16. Maciel MED. A equipe de saúde da família e o portador de transtorno mental: relato de uma experiência. Cogitare Enferm. 2008;13(3):453-6. 